\title{
Millisecond Pulsar Timing in Effelsberg
}

\author{
M. Kramer, O. Doroshenko, A. Jessner, R. Wielebinski \\ Max-Planck-Institut für Radioastronomie, Bonn, Germany
}

A.Wolszczan

Department of Astronomy and Astrophysics, Penn State University

F. Camilo

The University of Manchester, NRAL, Jodrell Bank, UK

J.H. Taylor

Physics Department, Princeton University

K.M. Xilouris

NAIC, Arecibo Observatory, Puerto Rico

\section{Introduction}

Millisecond pulsar as clocks are excellent tools for studying a variety of phenomena in physics and astrophysics (e.g. Foster \& Backer 1990). We have been observing millisecond pulsars with the 100-m Effelsberg radiotelescope since April 1994. Initially, the goal of this program was to help continuing the timing of Arecibo pulsars during the upgrade-related shutdown period of the 305-m radiotelescope. Gradually, the program has evolved to time and study the emission physics of all short period pulsars detectable from Effelsberg. In particular, polarization measurements are used to make inferences about the emission physics of millisecond pulsars (cf. Xilouris \& Kramer, this proceeding). At present, the programme involves approximately monthly observations of a set of 22 sources.

\section{Observing system}

The observations are made with a sensitive dual channel HEMT receiver, which is installed in the primary focus of the 100-m radiotelescope and is tunable in a frequency range from $1.3 \mathrm{GHz}$ to $1.7 \mathrm{GHz}$. For timing observations, centre frequencies of $1.41 \mathrm{GHz}$ and $1.71 \mathrm{GHz}$ have been chosen. The system has a temperature of $T_{\text {sys }} \sim 25 \mathrm{~K}$ and a gain of $1.5 \mathrm{~K} / \mathrm{Jy}$. The signals are passed through a four channel, $60 \times 666 \mathrm{kHz}$ filterbank and then postdetection dedispersed in hardware. The four outputs providing full polarization over $40 \mathrm{MHz}$ bandwidth are synchronously folded at the topocentric pulse period before a 15 s sub-integration is written to disk. Pulse arrival times are measured using a hydrogen maser clock which is routinely calibrated via time signals from the 

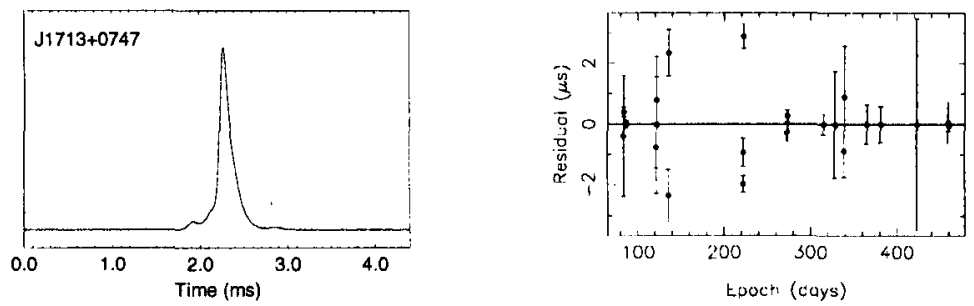

Figure 1. a) Profile and b) post-fit residuals obtained for J1713+0747 $(\mathrm{rms}=2.9 \mu \mathrm{s})$. Epoch zero is identified with MJD 49454 .
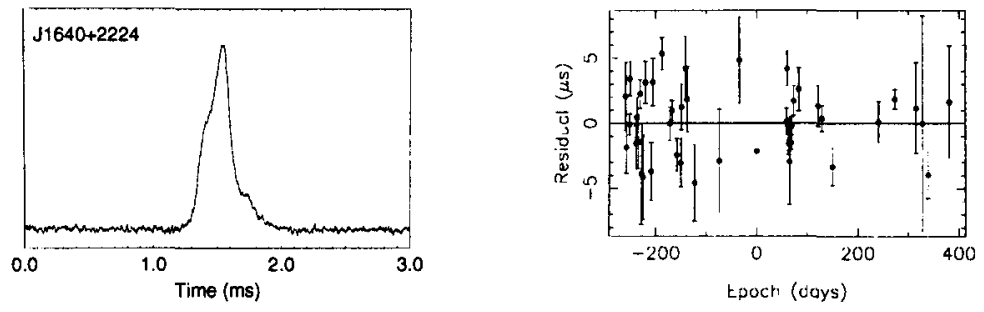

Figure 2. a) Profile and b) post-fit residuals obtained for combined Arecibo and Effelsberg data of $\mathrm{J} 1640+2224(\mathrm{rms}=2.5 \mu \mathrm{s})$. Epoch zero marks the beginning of Effelsberg observations at MJD 49454.

Global Positioning System of satellites (GPS). Gain control and flux density measurements are made using a switchable calibration signal.

\section{Data analysis and results}

The timing analysis was performed using the TEMPO programme (Taylor \& Weisberg 1989), as well as the independently developed code called TIMAPR (Doroshenko \& Kopeikin 1995). Examples of pulse shapes and timing residuals obtained from Effelsberg observations are shown in Figures $1 \& 2$. Further timing and polarization results will be published elsewhere (e.g. Xilouris \& Kramer, these proceedings; Wolszczan et al. 1996).

Acknowledgments. Arecibo Observatory is operated by Cornell University under cooperative agreement with NSF.

\section{References}

Doroshenko O.V., Kopeikin S.M. 1995, MNRAS, 274, 1029

Foster R.S., Backer D.C. 1990, ApJ, 361, 300

Taylor J.H., Weisberg J.M. 1989, ApJ, 345, 434

Wolszczan A., et al. 1996, in prep.

Xilouris K.M., Kramer M. 1996, these proceedings 